\title{
Pensamiento pedagógico colombiano: Martín Restrepo Mejía. Una mirada a sus conceptos de pedagogía, infancia, maestro y escuela ${ }^{1}$
}

\author{
Colombian pedagogical thought: \\ Martín Restrepo Mejía. \\ A glance at his concepts of pedagogy, childhood, teacher and school \\ Pensamento pedagógico colombiano: Martín Restrepo Mejía. \\ Um olhar sobre seus conceitos de pedagogia, infância, professor e escola.
}

\section{Absalón Jiménez Becerra ${ }^{2}$ \\ Universidad Distrital Francisco José de Caldas (Colombia)}

Recepción: 24/01/2017

Evaluación: 19/04/2017

Aceptación: 24/08/2017

Artículo de Investigación - Reflexión

DOI: https://doi.org/10.19053/01227238.7578

\section{RESUMEN:}

El artículo se enmarca en el inicio de una línea de investigación: Historia del pensamiento pedagógico colombiano. Esta propuesta busca rescatar el trabajo de una serie de intelectuales de la educación, quienes, por medio de sus razonamientos, intervenciones, conferencias, ensayos, y libros han aportado al ejercicio reflexivo de la educación local y regional. Esta primera investigación rescata la vida y obra de uno de los primeros intelectuales de la educación colombiana; Martín

1 Este artículo es el resultado de un capítulo de la investigación "Pensamiento Pedagógico Colombiano", financiado por el CIDC-UDFJC, código GI-PR-013-FR-020. La investigación aborda el rastreo de la vida y obra de pedagogos colombianos en el periodo comprendido entre 1870 a la actualidad. Se articula con el proyecto de "Educadores Latinoamericanos" de Grupo de Investigación "Historia y Prospectiva de la Universidad Latinoamericana". HISULA, avances en la Revista Historia de la Educación Latinoamericana. RHELA y la colección del citado proyecto marco.

2 Licenciado en Ciencias Sociales de la Universidad Pedagógica Nacional, Magister en Estudios Políticos de la Pontificia Universidad Javeriana, Magister en Historia de la Universidad Nacional de Colombia, sede Bogotá, D.C., es Doctor en Educación del acuerdo interinstitucional UPN, UniValle y Universidad Distrital de Bogotá. En la actualidad es Profesor de Planta de la Universidad Distrital Francisco José de Caldas de Bogotá, particularmente de la Maestría en Educación y del Doctorado Interinstitucional en Educación. Sus líneas de investigación se enmarcan en la historia social y política de los siglos XX y XXI en Colombia, y en la historia de la infancia, escuela y pedagogía, en el mismo periodo. Email: abjibe2012@hotmal.com 
Restrepo Mejía (1865-1935). Se enfatiza especialmente en sus reflexiones y representaciones de la pedagogía, la infancia, el maestro, y la educación escolar.

Palabras clave: pensamiento pedagógico; pedagogía; infancia; maestro; escuela; proyecto educadores HISULA.

\begin{abstract}
This article is part of a nascent line of research: History of Colombian pedagogical thought. This proposal aims to recover the work of a group of intellectuals of education; whose reflections, interventions, conferences, essays, and books have contributed to the reflective exercise upon local and regional education. This first research presents the life and work of Martín Restrepo Mejía (1865-1935), one of the first education intellectuals of Colombia. Especial emphasis is laid on his considerations and representations of pedagogy, childhood, teacher, and school education.
\end{abstract}

Keywords: pedagogical thought; pedagogy; childhood; teacher; school; educators project Hisula.

\section{RESUMO}

$\mathrm{O}$ artigo se situa no início de uma linha de pesquisa: Historia do pensamento pedagógico colombiano. Esta proposta busca resgatar o trabalho de uma série de intelectuais da educação, que por meio de seus raciocínios, intervenções, conferências, ensaios e livros contribuíram com o exercício reflexivo da educação local e regional. Esta primeira pesquisa resgata a vida e obra de um dos primeiros intelectuais da educação que a Colômbia teve: Martín Restrepo Mejía (1865-1935). Enfatiza-se especialmente suas reflexões e representações da pedagogia, a infância, o professor e a educação escolar.

Palavras-chave: pensamento pedagógico; pedagogia; infância; professor; escola; Projeto educadores Hisula.

Contextualización Idioma Maya
Kaqchikel
Contextualizadora: Myrna Lucy
Xajpot Sanain
Ministerio de Educación de
Guatemala

\section{RUK'U'X SAMAJ}

Re jun peraj samaj re ntikir rikin rucholajem rukanoxik na'oj: Rutzijol ch'ob'onïk pa ruwi rub'eyal tijonïk richin colombiano. Re jun na'oj re' nrajo chi nikol ri kina'oj ri yalan yechobon pa ruwi' tijonïk, ruma nkiya' kina'oj chupan ri kitzijonïk, ri kisamaj, ri kitz'ib'anik nkiya' richin yoj ch'ob'on pa ruwi' tijonik pa taq kitinamit akuchi e k'owi'. Chupam re jun nab'ey na'oj re, xkikanöj rukolik k'ik'aslem ri nab'ey taq chob'onela' pa ruwi ri tijonïk richin ri tinamït Colombia; Martín Restrepo Mejía (1865-1935). Janta pe niya'ox runataxik chupam kich'ob'oj chuqa' toq yech'o pa ruwi' rub'eyal tijonï, pa kiwi' ri ak'wala', pa kiwi' ri tijonela', chuqa' ri tijonïk pa tijob'äl jay.

Ruk'u'x tan tzij: na'oj richin rub'eyal tijojik; rub'eyal tijonik; pa kiwi' ak'wala'; pa kiwi' tijonela'; chupam ri tijob'äl; Na'oj samaj tijonela' Hisula.

\section{INTRODUCCIÓN}

El pensamiento pedagógico, en la presente propuesta de análisis, se puede definir como el conjunto de ideas por medio de las cuales se reflexiona en torno a la educación en la que están en juego un conjunto de representaciones por parte de un grupo de intelectuales de la educación 
de lo que significa: la escuela, los procesos de enseñanza y aprendizaje, la labor del maestro, la concepción del niño como sujeto educativo, como también el papel político que ha jugado la pedagogía en nuestro país.

El contexto teórico y conceptual en el que se ubica el pensamiento pedagógico colombiano, es la historia de la educación y la pedagogía ${ }^{3}$, debido a que nuestro interés es dar cuenta de la presencia de una serie de pedagogos nacionales, desde la tercera parte del siglo XIX hasta el tiempo actual. Creemos que, al contextualizar su labor y presencia en la historia de la educación colombiana, y rastrear y valorar su obra, realizaremos una investigación sobre el pensamiento pedagógico colombiano que se constituirá en un aporte a la pedagogía como disciplina fundante en el proceso de formación de maestros. En términos metodológicos la principal fuente de esta pesquisa de pensamiento pedagógico es la misma obra de Martín Restrepo Mejía, la cual ha sido poco valorada por la historia de la educación colombiana. Por lo demás, en la lógica de investigación histórica, su conocimiento es por naturaleza provisional e incompleto, aunque no por ello falso; selectivo, aunque no por ello arbitrario, y limitado y definido por las preguntas formuladas a las fuentes por parte del investigador ${ }^{4}$.

El pensamiento pedagógico colombiano se convierte en un tema importante de investigación en el marco de los programas de formación de docentes en el ámbito de pre-grado, maestría y doctoral. En ciertos espacios de estas unidades académicas, se evidencia el desconocimiento de la pedagogía colombiana, su devenir y transformación; los maestros, pedagogos e investigadores en formación, cuentan, en ocasiones, con una visión mucho más clara de la pedagogía y el papel de los pedagogos europeos y norteamericanos, que de las transformaciones que se han presentado en el ámbito regional y nacional. Frente a esta situación nos hemos planteado, de tiempo atrás, las siguientes preguntas: ¿Cuál ha sido el aporte de los pedagogos colombianos y su pensamiento en la constitución del campo intelectual de la educación?, ¿Qué caminos transitaron, en términos de su historia de vida - espacio biográfico-, para terminar abordando el tema de la pedagogía colombiana?, ¿Qué nivel de reconocimiento tienen los pedagogos colombianos en el ámbito nacional e internacional?, ¿Qué papel han jugado los pedagogos colombianos en la transformación del pensamiento pedagógico local?, ¿Cuáles son los principales ensayos, artículos y libros, por medio de los cuales dan a conocer sus percepciones e ideas pedagógicas? Y por último, ¿cuál ha sido su incidencia en la transformación de la escuela y en la política educativa colombiana?

La historia del pensamiento pedagógico, a través del estudio de las ideas y reflexiones académicas de los intelectuales de la educación, se debe realizar sin perder de vista el trasfondo ideológico y político en el que se encontraba inmerso el país en determinados periodos de nuestra historia. Como se dio a conocer en una anterior publicación, la constitución del Campo intelectual de la pedagogía en Colombia ${ }^{5}$, responde a una serie de acontecimientos en el que

3 Véase la Revista Historia de la Educación Latinoamericana. RHELA., dedicada en sus 27 números a un educador Latinoamericano.

4 Absalón Jiménez Becerra, "Algunos elementos para la investigación en historia". En Absalón Jiménez Becerra y Alfonso Torres Carrillo: La práctica investigativa en Ciencias Sociales Colombia. (Bogotá, D.C.: UPN, 2004), 138.

5 Absalón Jiménez Becerra, El campo de la pedagogía y otros ensayos sobre la historia de infancia en Colombia. (Bogotá, D.C.: UDFJC, 2014$), 19$. 
participan también los sujetos de saber e intelectuales de la educación. Las relaciones dentro del campo intelectual de la educación y la pedagogía, como sus relaciones con otros campos, expresan una particular lógica de dominación que se encuentra articulada a una serie de acontecimientos históricos, como conflictos de carácter social, movimientos sociales y culturales, el devenir de la restructuración y cambio del sistema educativo, además de las transformaciones de carácter político y cultural.

Inspeccionar el pensamiento pedagógico desde el aporte de sus principales intelectuales, en este caso Martín Restrepo Mejía, es inspeccionar la manera como la educación influye en la cultura y la mentalidad de un pueblo. Reconocer este sistema de pensamiento nos permite dar cuenta de lo que ha sido el proceso de formación de nuestra sociedad en cada periodo específico, de un conjunto de metas, algunas que se plantearon como probables y también otras que se lograron realizar.

\section{La vida y obra de Martín Restrepo Mejía}

Abordar la vida o el espacio biográfico ${ }^{6}$ de los intelectuales de la educación, es dar cuenta, en ocasiones, de un espacio eminentemente subjetivo. En el caso de Martín Restrepo Mejía, al inspeccionar los pocos detalles que se saben de su vida privada, valorar su labor pedagógica, su compromiso como maestro de escuela y, luego, como un intelectual de la educación destacado, ingresando de manera primordial por medio de la lectura de su obra, damos cuenta de la intención de rastrear su huella, la singularidad y trascendencia de su pensamiento pedagógico.

Martín Restrepo Mejía, nació el 2 de noviembre de 1861 en la ciudad de Medellín y falleció, casi en el olvido, hacia mediados de los años treinta del siglo XX. En realidad, no hay una fecha exacta de su muerte ni tampoco claridad del lugar en que murió. Aunque todo parece indicar, que falleció a los 74 años en la ciudad de Cali, en momentos en que realizaba su última labor pedagógica como directivo de un plantel. Fue hijo de Silverio Restrepo Fernández y Julia Mejía de la Torre; de su familia se sostiene que provenían de un sector humilde de la sociedad antioqueña y que, debido a los inconvenientes económicos, tuvieron que migrar de un lugar a otro en búsqueda de una mejor fortuna. ${ }^{7}$

De entrada, su origen social lo marcó; fue un hombre ajeno a la aristocracia política de la época y su situación de "liminidad", 8 al encontrase entre el adentro y el afuera, entre la incorporación y separación; entre la necesidad de

6 Franco Ferrarotti, "Las Historias de Vida como Método", Convergencia Vol. 14, No. 44 (2007): 17. Diana Elvira Soto Arango. La Escuela Rural en Colombia. Historias de Vida de Maestras. Mediados del Siglo XX. Tomo VI. Colección Educadores., (Tunja, Colombia: UPTC 2014). José Pascual Mora, Diana Elvira Soto Arango, José Rubens Lima Jardilino. La historia de la educación en América Latina: la contribución y aportes de la Sociedad de Histoira de la Educación Latinoamericana (1994-2014). História da Educação, Vol. 21 No. 51 (2017) 351-375. https://dx.doi.org/10.1590/2236-3459/66357

7 Un primer borrador de lo que fue la vida de Martín Restrepo Mejía, lo realiza Luis Antonio Bohórquez Casallas, en su libro Evolución Educativa en Colombia, publicado en 1956. En este libro destaca que no hay ningún pedagogo que haya influido tanto en la cultura del país en la primera mitad del siglo XX. Como maestro se destacó como el precursor católico de la escuela nueva, apagándose con él, una vez desaparece de la escena pública el apostolado de la docencia.

8 El antropólogo Arnold van Gennep, introdujo este concepto para describir la fase intermedia entre la separación e incorporación presente en los ritos de paso. Consultar: Arnold van Gennep, Los ritos de Paso. (Madrid, Alianza Editorial, 2008), 35. 
ser reconocido académicamente por parte de misiones religiosas de la Iglesia católica, sus intereses educativos y, particularmente, los conservadores que lideraron la regeneración del país entre 1878-1898. Restrepo Mejía, finalmente fue excluido de la política y la orientación educativa, que tomaría el país desde un enfoque liberal, en la década de 1920.

Los diferentes hechos educativos que acompañaron esta coyuntura, ante todo el Primer Congreso Pedagógico Nacional de 1917, trajo como resultado un desconocimiento de su obra, acompañado de un intencional olvido, producto de la intransigencia política de la época, que tuvo varias expresiones en lo religioso, lo político y lo educativo.

Según el investigador Oscar Saldarriaga Vélez, las razones por las cuales se olvidó la figura de Martín Restrepo Mejía, como pionero de la pedagogía en Colombia, son al menos tres: primero, la desaparición de las cátedras de historia de la pedagogía en el proceso de formación de maestros en Colombia a mediados del siglo XX; segundo, el gran impactó que tuvo Agustín Nieto Caballero (18891975) ${ }^{9}$, como pedagogo liberal, moderno y de avanzada, menospreciando a Restrepo Mejía por ser católico y conservador; y en tercer lugar, el proceso de secularización de la sociedad colombiana que deslegitimó a la Iglesia católica, su propuesta educativa y los intelectuales que la promovían ${ }^{10}$.

Su vida como maestro de escuela, inicia muy joven: a los quince años de edad se dedicó a la labor de maestro en el parvulario. Por diversas circunstancias, Martín Restrepo Mejía, ejerce primero la labor de maestro con pequeños niños en la ciudad de Medellín, aproximadamente desde 1876, año en que se encuentra en su punto más alto la "guerra civil de las escuelas".

De su vida como maestro podemos dar cuenta de que fue una persona dedicada al estudio y a la reflexión académica, y que desde el inicio de su labor luchó contra la adversidad y fue un comprometido del amor a la pedagogía, a la labor didáctica y a la maestranza escolar. Ser maestro, para Restrepo Mejía, se constituyó en un compromiso de vida, de interacción con las escuelas y los niños, de responsabilidad con la labor didáctica, con la práctica pedagógica, la reflexión educativa, la elaboración de manuales y el compromiso en el proceso de formación de educadores.

Mientras esto ocurre, la "guerra de las escuelas" en 1876, nuestro pedagogo en mención, Martín Restrepo Mejía, en 1878, se había trasladado a la ciudad de Buga, departamento del Valle, para fundar una pequeña escuelita con el deseo de trabajar y estudiar a la vez. Producto de esta experiencia ganada, luego, se trasladó a Cali y, en 1885, con su hermano Luis, dirigieron el Colegio de Jesús. En ese año, en un periódico bogotano, por casualidad, leen la invitación del Ministerio de Instrucción Pública, dirigido a los profesores del país, para escribir y presentar en un concurso público un tratado de pedagogía. El Ministro de la

$9 \quad$ Véase el número monográfico sobre Agustín Nieto Caballero y los principios de la Escuela Activa, en Revista Historia de la Educación Latinoamericana. RHELA, No. 5.139-143. Y Liliana Paternina Soto "Selección bibliográfica sobre Escuela Nueva en Colombia RHELA, No. 5, 144-148)

10 Oscar Saldarriaga Vélez, Del oficio de Maestro. Práctica y teorías de la pedagogía moderna en Colombia. (Bogotá D.C.: Cooperativa Editorial Magisterio, Grupo de Historia de la Práctica Pedagógica, 2003), 92. 
época, Dr. Enrique Álvarez Bonilla, ofrecía comprar el resultado del mejor libro con el fin de adaptarlo para la enseñanza en las Escuelas Normales.

Es de anotar que, frente a esta convocatoria, los hermanos Restrepo Mejía, habían ganado varios años de práctica en asuntos pedagógicos, pero no conocían los métodos modernos ni habían hecho un estudio especial de autores y asuntos pedagógicos, lo que, en principio, se constituyó en una desventaja. De acuerdo a las palabras de Martín Restrepo Mejía:

Le propuse a Luis escribir aquel libro. Él, que era modesto porque conocía mejor que yo nuestra ignorancia, se resistió por varios días. Comuniqué mis deseos a mi santa madre, y ella triunfó, como siempre en el ánimo de mi hermano.

Aquella noche, cumplidas las atenciones que nos imponían los alumnos internos, colocamos dos mesas unidas en la mitad del cuarto de Luis, y cada uno se puso a emborronar cuartillas de papel. Yo escribí una disertación sobre el niño; y otra, Luis sobre la educación. Cuando leíamos en voz alta nuestros escritos, comprendimos que no era así como se debía trabajar. Aquello no tenía sabor didáctico ni importancia técnica. Era preciso estudiar previamente la materia y formar un plan de obra ${ }^{11}$.

Frente a esta realidad, los hermanos Restrepo Mejía decidieron conseguir cuantos libros de filosofía y educación se podían obtener en la ciudad de Cali, empezando así una ordenada y juiciosa labor nocturna de lectura y escritura que demoró nueve meses de trabajo. El alma académica de los hermanos Restrepo Mejía se unió hasta identificarse y complementarse mutuamente. La labor en la escuela con sus estudiantes, les comprometía la jornada diurna y en las noches el compromiso con el principal manual de pedagogía que tendría el país desde el último tercio del siglo XIX hasta por lo menos 1920. En palabras de Restrepo Mejía:

Aquellas noches tienen en mi memoria el aspecto y la frescura de un manantial. Al cabo de nueve meses enviamos al Ministro de Instrucción Pública, con el pseudónimo de A. y B., una obra informe e incorrecta, que más contenía principios que reglas de educación. Devueltos de Bogotá los manuscritos por haberse prolongado el plazo del concurso, los rescatamos y los volvimos a enviar oportunamente... Mientras tanto corría ya el año 1887, mi hermano fue nombrado inspector de Instrucción Pública de la Provincia del Pacífico, cargo en cuyo ejercicio murió de insolación en los llanos de San Jerónimo. En mi dolorosa soledad seguí reformando nuestro libro y en 1888 obtuve parte del destacado éxito ${ }^{12}$.

Efectivamente, en el mes de mayo de 1888, en el marco del resultado final de un concurso convocado por el Ministerio de Instrucción Pública para los maestros del país, se dan a conocer los resultados, que destacan como ganador el libro de los hermanos Restrepo Mejía, Elementos de Pedagogía. Dicha obra ganadora,

11 Martín Restrepo Mejía, Labor didáctica. (Bogotá: Imprenta Moderna, 1909), 5.

12 Martín Restrepo Mejía, Labor didáctica, 5. 
escrita en provincia, se constituyó en el principal texto para la formación de maestros en las Escuelas Normales de Colombia. En su momento, se definió como una obra extensa, rigurosamente didáctica y escrita en lenguaje claro y castizo.

Martín Restrepo Mejía, entre 1892 y 1898, fue profesor de filosofía de la Universidad del Cauca en Popayán, que como centro científico del sur del país, lo acogió y valoró como un intelectual de la educación. En su estadía en la ciudad de Popayán, preparó, ajustó y publicó la segunda edición de Elementos de Pedagogía, en 1893, el cual se presentó como un libro de lectura útil y provechosa para la formación de maestros en las escuelas normales.

Para los conocedores del tema, los métodos pestalozzianos reivindicados por Retrepo Mejía, no podían convertirse de manera torpe en un arma de partido. Para nuestro pedagogo en mención, el niño debía ser pensado así, desde los preceptos de Pestalozzi, bajo un sano sistema pedagógico en el que la cruz de la civilización moderna debía sopesarse con la eficacia de la pedagogía cristiana.

En el departamento del Cauca lo nombraron Inspector General de Instrucción Pública y, luego, la gobernación lo nombró, durante cinco años, rector de la Universidad del Cauca. De hecho, siendo rector, combinó su actividad dictando clases de castellano en la Escuela Normal de Institutoras, en la ciudad de Popayán, lo que le implicó no solo enseñar la asignatura, sino también pensar la manera de enseñar lengua castellana. En el marco de sus cátedras, producto de su particular modo de ejercer la práctica pedagógica, y como formador de formadores, es que se dio a conocer, primero, a manera de folletos y luego el texto completo, en 1894, de Gramática de la Lengua Española, en la que buscó exponer con claridad y precisión todos los principios de nuestra lengua. Para Retrepo Mejía, dicha tarea la pretendió realizar: "Sin las arideces filosóficas de la Gramática del Señor Bello y sin aquella larga y fastidiosa enciclopedia de frases y vocablos viciosos, que puestos al frente de sus respectivas correcciones, llevan a la confusión de la mente de los alumnos"13.

La anterior fue una obra pensada para trabajar en los colegios, para el uso de los maestros y la aplicación con los estudiantes. Retrepo Mejía, como profesor de esta materia, proponía así, la enseñanza de la lengua castellana como prioridad en la escuela y en el proceso de formación de maestros normalistas. La obra del pedagogo y no del filólogo, llenaba un vacío con singular acierto y maestría para la instrucción de la juventud, escoger métodos adecuados para el análisis de funciones gramaticales que daba como resultado una regla general de aplicación práctica de la gramática, que se fijaba fácilmente en la memoria de los estudiantes. Luego, en 1907, dio a conocer tres libros en la ciudad de Bogotá; el primero, Aritmética para las escuelas, el cual fue producto de una larga reflexión y ejercicio de práctica pedagógica, que surgió desde el año 1885, cuando en la ciudad de Cali dio a conocer un primer folleto sobre números enteros; luego vino otra serie de folletos sobre números fraccionarios, números complejos y números

13 Martín Restrepo Mejía, Labor didáctica, 26. 
proporcionales. Dicho texto de matemáticas escolares, desde su inicio, buscó acercar al niño "de los fenómenos a la ley, del ejemplo a la regla, del análisis a la inducción; de la síntesis a la deducción y a la aplicación"14. Dicha propuesta expositiva de la matemática, se ubicó en los métodos de la pedagogía moderna, basada en iniciativas de carácter intuitivo, inductivo y deductivo, como era el parecer de la época. Este texto de matemáticas debía transmitir los conocimientos al estudiante, haciendo sencillo el aprendizaje en una materia abstracta, como lo era la aritmética.

El segundo libro, que dio a conocer en ese año, fue Geografía Universal, que se dividía en tres secciones: la primera, nociones fundamentales de geografía; según Restrepo Mejía, en esta se abordaba "lo que todo niño debía conocer hasta de memoria" y que hacía parte de una Geografía para las escuelas; en la segunda, el maestro encontraba noticias de diferentes países del mundo, que permitía ampliar la explicación y la información de la primera; y en la tercera sección, se encontraban datos numéricos y estadísticos, ordenados de tal modo que se lograban hacer interesantes comparaciones. Era un texto compuesto, que contenía información de uso escolar sobre geografía astronómica, geografía física y geografía descriptiva.

El tercer libro, que dio a conocer en 1907, Historia sagrada y eclesiástica en sus relaciones con la historia universal, nació por solicitud del gobernador de Cundinamarca, como un curso de enseñanza cíclica de la historia para las escuelas primarias. Es un libro de síntesis, en el que dio a conocer el devenir de la religión y la historia de la humanidad, con todas sus grandezas y horrores desde el momento del nacimiento de Cristo, hasta inicios del siglo XX en Colombia. Según Martín Restrepo Mejía, fue un libro pensado para los niños, pero con utilidad didáctica para los maestros.

Para Oscar Saldarriaga Vélez, nuestro pedagogo en mención, Martín Retrepo Mejía, "inaugura una tradición pedagógica que pensó el oficio del maestro como un trabajo de artista que trabaja sobre el alma de los alumnos utilizando un instrumento que nunca ha cesado de rondar la vida de los maestros: la fuerza del amor, una especie particular de amor, el amor pedagógico"15. Por el año de 1912, instalado ya en la ciudad de Bogotá, en la calle $16^{\mathrm{a}}$, entre carreras $6^{\underline{a}}$ y $7^{\mathrm{a}}$, antigua casa del General Santander, fundó la Escuela Martín Retrepo Mejía, la cual estuvo durante diez años bajo la administración de su esposa Elisa Arboleda; es decir, aproximadamente hasta el año 1922, momento en que migró a la ciudad de Tunja, para dirigir el Colegio de Boyacá, durando allí ocho años. ${ }^{16}$

En su estadía en la ciudad de Bogotá, para inicios de 1914, dio a conocer el libro: Pedagogía doméstica, dedicado a las familias, obreros e industriales, e inspirado en una serie de principios, como: autoeducación, educación de los hijos y dirección del hogar. Martín Restrepo Mejía, mediante este libro, pretendía incidir en la formación y condición moral de los obreros, campesinos y gentes humildes, luego

14 Martín Restrepo Mejía, Labor didáctica, 55.

15 Oscar Saldarriaga Vélez, Del oficio de Maestro. Práctica y teorías de la pedagogía moderna en Colombia, 94.

16 Por lo demás, como una anécdota de la historia, en el Colegio Martín Restrepo Mejía, el líder liberal Jorge Eliécer Gaitán (1898- 
de que Colombia venía de vivir los lastres de las guerras civiles y los defectos de una educación que arraigaba hábitos "perniciosos" para la mayoría de la población. Para Restrepo Mejía, en Colombia, la pedagogía doméstica se ubicaba en el discurso higienista de la época. Para este intelectual de la educación, el pueblo, lejos de respaldarse en las reglas de higiene alimenticia, solo consumía en determinadas zonas del país, chicha y coca, sustancias que adormecían el estómago e impedían despertar el apetito de alimentos necesarios para reparar la pérdida de los tejidos. Con esta alimentación, los individuos y la raza se encaminaban inevitablemente a la ruina y al desastre. En sus palabras:

Da grima ver en las poblaciones más importantes de la República, hombres, mujeres y niños con vestidos mugrientos, con manos, pies y cara, que producen repugnancia. No basta la pobreza para explicar esos repugnantes focos de infección: uno puede ser pobre y vestir con limpieza. Para hallar el verdadero motivo de tamaña injusticia hay que buscarlo en la falta de educación, en la decidia, en la carencia de constantes correcciones por parte de quienes están al frente del pueblo ${ }^{17}$.

Encontramos así, en esta propuesta, cierto tipo de educación pensada para la intervención de la población y cierto tipo de educación popular en la que se reivindicaba desde una percepción católica de la época, el respeto al cuerpo, la higiene corporal, la importancia del ejercicio y la educación física; la reivindicación de una instrucción sobria, metódica y proporcionada a las inteligencias del niño, del joven y el adulto, por parte del maestro. En la Pedagogía doméstica, los maestros debían atender el cultivo de la memoria y la imaginación; la conciencia, formada por la buena educación; la disciplina, basada en buenos hábitos, y la obediencia del obrero.

Sin embargo, las tensiones y cuestionamientos hacia la figura de Martín Restrepo Mejía, se empezaron a presentar en 1917, en el marco del Primer Congreso Pedagógico Nacional, reunido en Bogotá, bajo el auspicio del Ministerio de Instrucción Pública, evento que se proyectó con el fin de conjurar la crisis de las escuelas normales, enfrentar el problema de la formación del magisterio y lanzar una nueva propuesta de modernización del sistema escolar. Todo parece indicar, que las conclusiones y decisiones políticas del Primer Congreso se dieron en contravía de la propuesta pedagógica, de los manuales y las iniciativas educativas de Martín Restrepo Mejía ${ }^{18}$. Los Hermanos Cristianos, quienes representaban un sector importante en dicho evento, en términos de las iniciativas educativas reformistas, cuestionarían los manuales y la producción intelectual de este pedagogo.

1948), cursaría el último año de bachillerato y se graduaría en 1919, para luego ingresar a estudiar Derecho y Ciencias Políticas en la Universidad Nacional de Colombia.

17 Martín Restrepo Mejía, Pedagogía doméstica. Autoeducación, dirección del hogar y educación de los hijos. (Bogotá: Editorial Barcelona, 1914), XXI.

18 Absalón Jiménez Becerra. "I Congreso Pedagógico Nacional Colombiano de 1917. Una mirada a sus tensiones y avances, cien años después de su realización”. (Revista Pedagogía y Saberes, No 48. Bogotá, D.C. CIUP-UPN). 
El ambiente académico y político se mostró adverso a Restrepo Mejía, por lo menos desde 1920, lo que trajo como resultado su marginamiento inicial, su desconocimiento académico, para llegar casi a su olvido total a mediados del siglo XX. De hecho, para 1909, en momentos en que daba a conocer una compilación de pronunciamientos y reconocimientos a su trabajo, en Labor didáctica, se había presentado ya, el primer cuestionamiento, debido a que el noventa por ciento de libros enviados a las escuelas públicas eran de su autoría. Esto causó tensión y recelo por parte de otra serie de sectores, ante todo religiosos, que trabajaban en la formación de docentes y, particularmente, en la educación de los niños en las escuelas del país, tanto a nivel urbano como rural.

En 1930, se dirigió a la ciudad de Cali, para regentar el Colegio Santa Librada y trabajar allí durante dos años. A finales de 1933, apareció un artículo de Restrepo Mejía, titulado: "Etapas de la civilización", en la Revista Colombiana, fundada por el entonces joven conservador, Laureano Gómez.

Aunque hubo una intención de condenarlo al olvido, cuando se reconstruye la historia del pensamiento pedagógico colombiano, evidenciamos que fue un maestro con un alto nivel de reconocimiento en la época de la Regeneración y hasta, por lo menos 1917, año en que se destacó como promotor y organizador del Primer Congreso Pedagógico Nacional. Fue un maestro y pedagogo, que marcó el devenir de la educación en Colombia, modernizó los conceptos de infancia, maestro, escuela, pedagogía y educación, transversales en el proceso de formación de docentes, e incidió por medio de sus manuales, en la labor didáctica y en las prácticas pedagógicas de la época.

A pesar de cierta situación de "liminidad", de estar entre el afuera y el adentro de los sectores políticos de la época y de los sectores educativos en pugna por la modernización del país, en términos educativos, toda su obra, acompañada de una serie de giros y transformaciones, se movía en un concepto moderno de escuela, acompañado de un concepto liberal de pedagogía que, se mantendría desde su reflexión inicial, en 1888, hasta su última obra, Pedagogía de párvulos, dada a conocer hacia 1925. Es decir, aunque conservador en su militancia política, fue un hombre abierto en su pensar educativo, humanista y liberal en términos pedagógicos.

\section{El concepto de pedagogía en Martín Restrepo Mejía}

Para Martín Restrepo Mejía, la pedagogía en la modernidad, debía estudiar las leyes del desarrollo humano para intervenirlo y perfeccionarlo. La pedagogía necesitaba de la enseñanza para educar al hombre de acuerdo a las leyes de la naturaleza, buscando fortalecer las potencialidades humanas, como lo eran: la percepción sensitiva, la intelectual y el raciocinio. La pedagogía no podía pensarse independiente a un sistema pedagógico, visto como el conjunto de principios supremos relativos a la naturaleza, estado actual y fin del hombre, a los cuales se subordinan las leyes del desarrollo humano y todos los trabajos del educador. En sus palabras, la pedagogía se definía como: 
La ciencia que estudia el desarrollo humano investigando su punto de partida, sus leyes y su fin, y arte que enseña los procedimientos más adecuados para educar al hombre según aquellas leyes. La Pedagogía al definirse mitad ciencia conviene con las demás ciencias antropológicas en que estudia al hombre, pero difiere de ellas en que le estudia en cuanto es un ser libre que se desarrolla. La parte de la Pedagogía que estudia las leyes del desarrollo humano es verdadera ciencia; más la que enseña los procedimientos más adecuados para educar al hombre según aquellas leyes, es un arte $^{19}$.

En el libro Elementos de pedagogía, en su primer párrafo, se nos recuerda, además, que la pedagogía viene de las voces griegas: paidós que significa niño; y agogos, que significa conductor, ayo. Los griegos y los romanos le daban el nombre de pedagogo al esclavo encargado de conducir al niño a las escuelas públicas, de cuidarle, ver por sus buenas costumbres y no abandonarle un instante en la casa paterna. Sin embargo, ya en la modernidad, la labor del maestro debía ser pensada desde los principios básicos de las doce leyes pedagógicas que rigen la naturaleza del acto educativo:

La primera ley pedagógica consiste en que el educador debe estudiar las leyes que rigen la naturaleza humana, respetarlas y facilitar su cumplimiento, principio que es basado en Santo Tomás: El arte debe imitar a la naturaleza; la segunda es que las potencialidades humanas deben desarrollarse ordenadamente, esto por grados sucesivos de manera que antes de trabajar en el desarrollo de cualquiera de ellas se alcance el desarrollo de las inferiores: educar la percepción sensitiva antes que la intelectual y esta antes que el raciocinio, principio promovido por Pestalozzi. La tercera ley, debe dejarse a la naturaleza el primer grado de desarrollo de las facultades, para no obligar a la naturaleza del niño a ejecutar actos para los cuales no está aún preparado. El trabajo intelectual prematuro produce cierto brillo engañador que alucina a los padres y maestros y que se paga luego con algo de anemia intelectual. La cuarta ley, establecida por Froebel, consiste en que toda educación y toda enseñanza debe ser en un principio indulgente, flexible y blanda; debe limitarse a proteger y vigilar sin propósitos previos ni sistema preconcebido; la quinta ley ordena que la educación sea completa o integral, esto es, que dirija todas las facultades del niño y que las dirija a todos los fines que sea preciso alcanzar. La sexta ley, dispone que en cada uno de los grados de la educación se atienda a perfeccionar los anteriores y a preparar los siguientes. La séptima ley, promulgada por Froebel, consiste en que la educación para que sea eficaz, debe darse cuidadosamente desde el principio de la vida del niño. Aquel gran pedagogo observó que la educación escolar es ineficaz si la procede o acompaña una viciosa educación doméstica; la octava ley pedagógica se funda en el principio de que la educación es libre y consiste en que todo trabajo educativo principia por inspirar al alumno el deseo de ejecutarlo, se debe respetar la curiosidad y el interés del niño. En todo trabajo educativo debe procederse de lo sencillo a lo difícil, de lo simple a lo compuesto, de lo conocido a lo desconocido, de lo concreto a lo abstracto. La novena ley, debe aprovecharse para la educación todo lo que interese a la curiosidad del niño y

19 Martín Restrepo Mejía y Luis Restrepo Mejía, Elementos de Pedagogía. (Bogotá: Imprenta Eléctrica, 1905) 1-2. 
en la que en los primeros años el juego debe ser la forma general de la educación. Esta ley reconocida por Froebel, establece que la alegría da salud y animación al cuerpo y al alma. La décima ley, dispone que el maestro dé el ejemplo para la consecución de todo fin que se desea obtener. La undécima ley, promulgada por Pestalozzi, consiste en que la educación se dé por aquella clase de trabajo que constituya la vocación natural del individuo. La doceava ley, establece que la duración del trabajo debe ser proporcional a la edad y demás condiciones del alumno y el descanso debe consistir ya en el cambio de una ocupación por otra, ya en la suspensión total de toda tarea. El descanso es un medio poderoso de desarrollo, porque permite la reparación de las fuerzas perdidas en el trabajo, siempre que no sea excesivo porque en tal caso enerva ${ }^{20}$.

De tal manera, en Elementos de pedagogía, hay una lectura integral de una serie de pedagogos modernos, entre ellos Pestalozzi y Froebel, mediados por la visión ontológica de Santo Tomás, en la que sin desconocer la tensión entre fe y razón, había espacio para una visión cristiana de educación. Las leyes pedagógicas respondían a unos principios básicos de época, cuyo interés era modernizar el discurso pedagógico acompañado de una serie de enunciados que buscaban incidir desde finales del siglo XIX en la política de formación de maestros que regía en las escuelas normales de la época.

Este manual, que orientó la formación de docentes en las Escuelas Normales, por lo menos hasta 1920, reivindicó al niño como preocupación central de la pedagogía moderna. En términos de práctica pedagógica, toma cuerpo la "positivización" del niño en el discurso pedagógico colombiano, respondiendo a la revolución pedagógica vivida a inicios del siglo $X X$, al proceso de "psicologización" del niño y "pedagogización" del mismo.

El niño, en la pedagogía moderna, debía ser pensado bajo principios básicos de potencialidad humana, su grado de desarrollo y la proporcionalidad en el tipo de trabajo intelectual que se establece en la escuela. La pedagogía, desde entonces, y de acuerdo a esta perspectiva, es pensada bajo un principio indulgente, amoroso y flexible en el mismo acto educativo. Dicha pedagogía se basaba en los principios de interés, espontaneidad y el ambiente de libertad que debía garantizar la escuela moderna, propuesta que se buscaba instalar en la formación de maestros normalistas en Colombia.

\section{Hacia un concepto de infancia moderna}

Al inspeccionar la obra de Martín Retrepo Mejía, logramos dilucidar un concepto de infancia, que se desprende de la lectura que hace de los pedagogos y discursos pedagógicos modernizantes de la época; y en segundo lugar, un concepto de niño, que se desprende de las prácticas de educación doméstica y educación escolar, cuyo punto de encuentro es la disciplina que, vista como

20 Martín Restrepo Mejía y Luis Restrepo Mejía, Elementos de Pedagogía, 135. 
ortopedia educativa, se mostraba como requisito previo a la posibilidad de gobernarse así mismo, a la conquista de la libertad.

El concepto de infancia, que se desglosaba de Elementos de pedagogía, en 1888, de manera paulatina se buscó instalar en las escuelas normales del país al convertirse en el principal manual de formación. Dicho concepto, moderno para su época, se basaba en las lecturas que los pedagogos colombianos habían hecho, desde la reforma educativa de 1870, de Jean Jacques Rousseau (17121788), Johann Heinrich Pestalozzi (1746-1827), Friedrich Froebel (1782-1752). Sin embargo, en Elementos de pedagogía, se debía tener en cuenta el abordaje crítico que Restrepo Mejía hacía de la obra de J.J. Rousseau, a quien comentaba con reparos al ubicarlo en el iusnaturalismo racionalista, que intentaba suprimir la ley divina en el proceso educativo. ${ }^{21}$

Para los hermanos Restrepo Mejía, la virtud nola podía alcanzar una educación excesivamente racionalista, objetivista y apartada de Dios. La educación natural, como la planteaba J.J. Rousseau (1712-1778), podía convertirse en ineficaz:

Un niño abandonado a las influencias de la naturaleza física y privado de toda sociedad con sus semejantes, podría alcanzar cierto desarrollo de sus fuerzas físicas y sensitivas, pero se verían sacrificadas las intelectuales y morales ${ }^{22}$.

No obstante, la anterior tensión con la obra de Rousseau, el concepto de infancia de Martín Restrepo Mejía, estuvo presente en sus reflexiones pedagógicas desde el momento mismo en que dio a conocer con su hermano Luis, Elementos de pedagogía. En este primer libro de pedagogía colombiana, nos aclara que la palabra infancia designó desde tiempos primitivos aquel periodo de la vida en que el niño no habla, comprobándolo así su etimología (in- negación; fari-hablar); ese periodo es poco más o menos, el de los dos primeros años de edad. Después se aplicaba a todo el tiempo en que el niño, aunque hablaba, no lo hacía con el discernimiento necesario, esto era, hasta los siete años; pero esta representación se podía extender hasta los diez u once años, periodo que se consideraba próximos a la infancia.

En Elementos de pedagogía, la infancia era reconocida como un sujeto activo, portador de un conjunto de potencialidades, como la percepción, la potencialidad sensitiva, la intelectual y el raciocinio. La infancia era vista desde entonces, como sujeto activo, en el cual se debía propender por su perfección de acuerdo a un proceso educativo, liderado por el maestro, pero ajustado a sus transformaciones físicas y psicológicas. Dicha infancia debía gozar de una educación completa, integral y flexible; desde un inicio, la educación escolar debía complementarse de una educación doméstica, acorde a un conjunto de intereses mutuos.

21 Los reparos a la obra de J.J. Rousseau, en el Emilio, lo mantuvo Martín Restrepo Mejía a lo largo de su vida académica, buscando inclusive establecer un "empalme" más claro con otros pedagogos modernos, como Pestalozzi, Froebel, María Montessori y Decroly, entre la anterior educación tradicional, vista como educación memorista y la "enseñanza activa". Lo anterior se puede consultar en su último libro, Pedagogía de Párvulos, obra publicada a mediados de la década de 1920.

22 Martín Restrepo Mejía y Luis Restrepo Mejía, Elementos de Pedagogía, 10. 
La propuesta educativa para la infancia moderna, fomentada por Martín Restrepo Mejía, debía ser proporcional a la edad del niño, reconociendo de entrada en este a un sujeto curioso, para quien el juego se constituía en su principal actividad, con potencialidades educativas inmensas.

En su práctica pedagógica, en sus reflexiones y elaboración de manuales educativos, Martín Restrepo Mejía, había individualizado constantemente a la infancia desde una perspectiva moderna. En una de sus últimas publicaciones, Pedagogía de Párvulos, ${ }^{23}$ dada a conocer a mediados de la década de 1920, evidenciaba la importancia del niño parvulario en Colombia. De tal manera, aclaraba que la escuela de párvulos hacía referencia a los establecimientos que solamente recibían niños de tres a siete años de edad, y se proponía prepararlos para las escuelas primarias, despertando y encauzando sus facultades.

En Pedagogía de párvulos, hace un recorrido de la "escuela memorista" o método tradicional de enseñanza, debido a que la formación de maestros en métodos activos aún en el país era nula, lo que generaba la necesidad de hacer un balance de los principales defectos en el método en que ellos fueron formados. Las horas de asistencia a la escuela para el niño parvulario, variaban según el clima y las costumbres de la localidad, pero, en todo caso, se sugería que los trabajos intelectuales se hicieran en la mañana, y la tarde se destinara a juegos y tareas sencillas.

A la primera sección del parvulario, se le educaba con juegos educativos, conversaciones y lecciones sobre juegos materiales. Al parvulario superior se le enseñaba a leer y escribir y se le darían lecciones orales de historia sagrada y patria, cálculo y geografía. Sugirió que la educación del parvulario estuviera a cargo de maestras, debido a que los niños pequeños requerían con frecuencia un cuidado material que ellas, por lo general, podía facilitar. A las maestras formadas en el método memorista le hacía ver que:

La maestra ha de tener sobre los pároulos un ascendiente natural que le haga querer, respetar y obedecer de los niños sin necesidad de represiones severas y mucho menos castigos. Ha de serles tan simpática que aún por estar con ella, quieran ir a la escuela. Si no lo consigue debe dejar el puesto ${ }^{24}$.

La maestra del parvulario debía considerarse y conducirse en la sección inferior como madre de sus alumnos. En la sección superior debía preocuparse por acostumbrar a los niños al orden y regularidad de los trabajos. De esta manera, se iba así avanzando a una disciplina más compleja, que comprometería luego a los niños en la escuela primaria. Las instituciones dedicadas al parvulario se distinguían de las cunas y los asilos, en que estos eran establecimientos de caridad para atender ante todo las necesidades materiales de los niños excluidos

23 El título completo del libro de Martín Restrepo Mejía, es Pedagogía de Párvulos. Exposición de la enseñanza activa, del cual no se tiene una fecha exacta de publicación. Creemos que la Editorial de Cromos, lo dio a conocer a mediados de la década de 1920, porque hay referencias claras de María Montessori y Ovide Decroly y su incidencia en la pedagogía colombiana.

24 Martín Restrepo Mejía, Pedagogía de párvulos. Exposición de la enseñanza activa. (Bogotá: Editorial de Cromos, ca.1925), 42. 
o pobres. El parvulario era una propuesta educativa que se centraba de manera universal en la primera infancia y que preparaba al niño de manera pedagógica a su incorporación formal a la escuela primaria, hacia los siete años de edad.

La pedagogía de párvulos se mostraba como un adelanto a mediados de la década de 1920, debido al avance de la antropología y psicología experimental, pero principalmente, a las ideas de los grandes pedagogos que en ese momento hacían parte de la escuela activa, entre los cuales, Restrepo Mejía, ya había destacado a Pestalozzi y Froebel, agregando para inicios del siglo XX, la incidencia que tenían en nuestro país María Montessori (1870-1952) y Ovide Decroly (1871-1932). Al descubrir la educación del parvulario, para Restrepo Mejía, estos pedagogos habían ajustado el método antiguo, cuyo principal defecto era un tipo de enseñanza que había conducido al memorismo y la pasividad del niño, sin preocuparse por la preparación y el desarrollo de las facultades infantiles.

La escuela moderna o "enseñanza activa" se proponía aprovechar el interés que todo niño tenía de aprender a hacer algo, acompañado de su curiosidad. La representación de infancia en la práctica pedagógica colombiana, terminó de modernizarse cuando se fomentó la educación del párvulo, que buscaba en el fondo aprovechar la más tierna edad del niño para fortalecer sus facultades sensitivas, intelectuales y motrices.

Las anteriores reflexiones son complementadas en el libro Pedagogía doméstica, dado a conocer en 1914. El niño, pensado desde el ángulo de la "educación doméstica", se convertía en un rayo de luz que aparecía en el hogar, siendo objeto de cuidado, abrigo y calor; además, se convertía en una persona que demandaba en los primeros meses, aseo, lactancia adecuada y cuidado en la alimentación, hasta llegar al destete.

Para inicios del siglo XX, de acuerdo a la mirada de Restrepo Mejía: "los castigos físicos eran indispensables en la primera niñez, cuando aún no tiene el niño freno interior de la conciencia, para evitar que se formen hábitos que impidan la buena dirección de la voluntad, cuando esta se potencia y se desarrolla. En este sentido, se debía contralar la insolencia del niño, sin embargo, los castigos no se debían realizar con ira, ni debían ser muy frecuentes" 25 .

Luego, en el niño de los tres a los siete años, al conocimiento sensitivo de las cosas que poseía, se le debía agregar el conocimiento intelectual. En este periodo, el niño ya era capaz de ideas generales, de comparar las cosas unas con otras, encontrar sus diferencias y semejanzas. A esta edad, el niño podía formar juicios sencillos y elementales sobre el orden físico y moral. La voluntad del niño, en esta etapa, aunque es muy débil, todavía se expresaba por medio de la curiosidad y el deseo de examinarlo y saberlo todo. Las múltiples preguntas que hacían los niños se debían contestar por parte de los adultos con verdad y tino.

En cuanto su educación intelectual, se debían desarrollar sus facultades con base en los sentidos y luego la percepción y el juicio. Restrepo Mejía aclaraba que no es de esta etapa el raciocinio, que se manifiesta aun débilmente; por

25 Martín Restrepo Mejía, Pedagogía doméstica. Autoeducación, dirección del hogar y educación de los hijos. (Bogotá: Editorial Barcelona, 1914), 142. 
consiguiente, nada de libros ni enseñanza de memoria, a excepción de unas oraciones y composiciones poéticas muy sencillas e inteligibles. Por medio del juego, se debía ejercitar la vista y el tacto. Por otro lado, la fantasía, vista como la capacidad de retener y reproducir las imágenes de las cosas percibidas y combinadas entre sí, era una facultad que demanda educación. Para educar la fantasía se debía hacer que el niño observara un objeto y, luego, sin tenerlo presente, diera los rasgos y cualidades principales, para luego exigirle que describiera un objeto que él se imaginara, junto a cualidades de varios ya observados.

A los cinco años, se le debía facilitar al niño un lápiz y pedazos de papel para iniciar el trazado de líneas y garabateo, y de manera paulatina, el niño pasaba luego al dibujo de objetos; el maestro debía enseñar en ese momento las vocales para luego enseñar las consonantes. En términos de la educación doméstica, Restrepo Mejía aconsejaba a los padres de familia, en esta etapa de la vida del niño:

No dejen sin freno la ira de vuestro hijo, porque ella produce la injuria, la calumnia, la maldición, la blasfemia, el asesinato, el suicidio. El niño bravo, impaciente, susceptible y pendenciero está en peligroso camino, del cual hay que apartarle a todo trance. La ira es una pasión defensiva. Es la relación de nuestra naturaleza sensible contra el mal que nos amenaza o alcanza. Tiene pues un fin útil y no hay que pensar en ahogarla; antes bien, debe despertarse en los niños tímidos y cobardes, porque sin ella queda el hombre inerme ante los peligros físicos ${ }^{26}$.

Para Restrepo Mejía, el niño, desde los seis o siete años, debía ir a la escuela. $\mathrm{Su}$ educación requería ya un trabajo continuo y sistemático, que era muy difícil de desarrollar en casa, a menos que los padres pudieran dedicarse a él exclusivamente. Además, la escuela tenía la ventaja de que iniciaba en el niño la necesidad de trabajar con las demás personas como parte del orden natural de la vida. Hacia los siete años se iniciaba el periodo de uso de la razón, que duraba hasta los diez u once años. En este periodo se conocía el orden moral, aunque no en todos sus detalles; el niño observaba no solo los fenómenos externos, como lo hacía en el periodo anterior, sino los internos y reflexionaba sobre ellos, como sobre los actos de los demás hombres.

En el periodo siguiente, el periodo próximo a la libertad, que iba hasta los trece o catorce años, el entendimiento era capaz de abstracciones y raciocinios más complicados. En este periodo, era importante enseñarle al hijo la higiene, saber conseguir las cosas necesarias para la vida, lo que implicaba aprehender a trabajar arte, industria y ciencia. En este periodo, la educación cognoscitiva se enlazaba con la educación intelectual, en cuanto debía acostumbrarse al niño a observar bien el mundo sensible, a fin de que eso le sirviera de base para inducciones y raciocinios. Entre los siete y los catorce años hay una preocupación

26 Martín Restrepo Mejía, Pedagogía doméstica. Autoeducación, dirección del hogar y educación de los hijos, 172. 
central que le compete a la educación doméstica y a la educación escolar, como lo era la disciplina.

La disciplina era vista por Restrepo Mejía, como el conjunto de medios del cual se valían los padres y el maestro, para que la voluntad del niño se acostumbrara al orden.

Los medios de que se valía la disciplina para obtener dicho fin, eran tres: la ley, la regla u orden, que prescribía la vida del niño; la autoridad, con que se imponían estas órdenes; y los estímulos, con que se excitan la voluntad del niño. Los medios debían emplearse de modo tal que tendiera a que en el niño se acomodase gustoso y convencido a las condiciones de la orden a fin de que poco a poco fueran innecesarios, tanto la autoridad paterna y los estímulos y fuera capaz de gobernarse así mismo, por convicción y voluntad recta. La disciplina que de esta manera y con este propósito procedía era la que se llamaba educativa ${ }^{27}$.

La disciplina educativa se diferenciaba de la disciplina social, que buscaba la conservación de los cuerpos colectivos y se implantaba donde la autoridad no era educadora, sino mas bien reguladora de los actos externos, como la sociedad civil, las corporaciones y el ejército. La disciplina educativa consideraba el orden como fin; la disciplina social, como medio para alcanzar otros fines. La disciplina educativa buscaba la perfección moral del individuo; la social, el orden necesario para que la comunidad se dirigiera convenientemente a su objetivo.

En cuanto a la autoridad, Restrepo Mejía, en Educación doméstica, aclaraba que los padres debían ejercer su autoridad con firmeza y dulzura. Bastaba la firmeza para la autoridad social, pero en el hogar donde la autoridad era educadora, se debía mezclar con el corazón y la cordialidad de los padres.

Lo que importaba en la educación moral es el amor a la ley que se obedecía, porque de este modo se ponía al niño en el camino de saber y querer gobernarse así mismo que era el punto en que la educación se daba por terminada. El gobierno, el self government, que dicen los ingleses, es el fin de la educación moral ${ }^{28}$.

Para Restrepo Mejía, si el niño era bien dirigido en los periodos anteriores, cuando llegaba a los catorce años de edad, estaba casi completamente terminada la educación de la potencia vegetativa, de la sensitiva y de la motriz, respecto de las cuales bastaba velar para queno se perdiera eléxito obtenido. Una vez conquistado el entendimiento, sabe observar, analizar, sintetizar, inducir, discurría con acierto y con vigor sobre los asuntos relativos a la materia y sus leyes. El entendimiento funcionaba como conciencia y como memoria con bastante claridad, ante todo, como entendimiento especulativo mejor que como práctico.

Así desde los catorce años, poco más o menos, debía ser dirigido el joven de manera que empezara a gobernarse así mismo, si daba muestra de que era capaz

27 Martín Restrepo Mejía, Pedagogía doméstica. Autoeducación, dirección del hogar y educación de los hijos, 88.

28 Martín Restrepo Mejía, Pedagogía doméstica. Autoeducación, dirección del hogar y educación de los hijos, 191. 
de hacerlo con recto criterio y energía suficiente. De manera paulatina, se debía ir soltando las riendas, controlando que el joven no abusara de la confianza ganada, con el objetivo de convertirse luego en un hombre de honor. Llegando así el día en que el joven se gobierne así mismo, sin el control de mundo adulto, sin el control de los padres y el maestro.

El concepto de infancia desarrollado por Martín Restrepo Mejía, con base en sus reflexiones en torno a la pedagogía moderna, las leyes pedagógicas en las que se ubica un conjunto de autores que habían reconocido las diferencias cualitativas de este sujeto con relación al mundo adulto y su reconocimiento como sujeto activo en la educación, se termina de complementar con sus reflexiones en torno a la pedagogía de párvulos, constituyéndose en uno de los pedagogos colombianos que descubre y reconoce a la primera infancia, particularmente la "infancia parvularia", vista como aquella que se encontraba preparándose a ingresar a la escuela primaria, en un rango de edad entre los tres y seis años.

Por otra parte, el concepto de niño que se desprende del "dispositivo de alianza", ${ }^{29}$ entre la familia y la escuela, entre la educación doméstica y la educación escolar, entre el padre de familia y el maestro, pasaba por unas prácticas de cuidado, abrigo y calor en la primera etapa, acompañadas de prácticas de "disciplinamiento" familiar que direccionaban su buena voluntad, orientaban su conocimiento sensitivo, percepciones y representaciones del mundo, agregando el conocimiento intelectual de las cosas. En el niño, la educación escolar, continúa y sistemática, debía ser el complemento ideal de una buena educación doméstica. La disciplina aplicada en torno a él, utilizaba como medios principales, las normas, la autoridad y los estímulos. Desde la perspectiva de Martín Restrepo Mejía con relación a los temas de infancia y su proceso educativo de nada servía una buena educación escolar si la educación doméstica era un fracaso. Esa infancia sensitiva que conquistaba percepciones en su proceso de formación vivía una serie de transformaciones cualitativas que el mundo adulto y la sociedad colombiana debían valorar de manera científica con base en los aportes de la pedagogía.

\section{Un nuevo tipo de maestro}

Para Martín Restrepo Mejía, la educación memorista o "educación tradicional", había formado un tipo de maestro que desconocía los avances de la pedagogía, el peso que tenía la psicología en el proceso de individualización del niño, sus transformaciones cualitativas, el peso que tenía las sensaciones, percepciones y representaciones para sacar adelante la pedagogía objetiva $\mathrm{y}$, en general, el reconocimiento del niño como sujeto activo en el proceso educativo. El nuevo contexto demandada la formación de buenos maestros, quienes debían ser

29 El dispositivo de alianza hace referencia al lazo entre estas dos instituciones, cuyo objetivo fundamental es la domesticación y el disciplinamiento final del niño. El dispositivo de alianza se ubica en una posición estratégica dominante, ya que se supone siempre una intervención en relaciones de fuerza y se inscribe en un juego de poder. Dicho concepto se desprende de Michel Foucault, Historia de la sexualidad I. La voluntad de saber. (México: Siglo XXI, 1976). 
ejemplo de su conducta, amantes de su tarea, dulces a la vez que firmes con los niños, atentos con los padres, hombres de estudio y buenos ciudadanos. Los maestros modernos debían desarrollar, fortalecer y fecundar en la infancia su raciocinio y capacidad intelectual.

La labor del maestro, al igual que el de educación, debía ser pensada en el marco de los medios de la educación intelectual. Para Restrepo Mejía, son tres los grandes medios de la educación intelectual: la enseñanza, la práctica y el medio social. En su concepción de maestro, hace énfasis especial en la enseñanza, que es el medio principal de la educación del entendimiento. La enseñanza servía para ejercitar y desarrollar las fuerzas intelectuales, como también para comunicar la instrucción. En sus palabras:

La enseñanza debía ser gradual y proporcionada, lo que quería decir que se debía acomodar al desarrollo intelectual del niño, sin violentarle, exigiéndole aprendizajes de materias que por su número, amplitud o su dificultad superen sus fuerzas. La enseñanza debía ser clara, pero la claridad varía según la edad del niño, lo que era claro para un joven podía ser inteligible para un párvulo. La enseñanza debía ser cíclica, la cual consiste en que los diversos grados de la enseñanza se diferenciaran por la amplitud, un número de pormenores con que la materias se da en los grados superiores, la enseñanza cíclica hace que el niño contemple todo el campo que abarca cada materia y que principiando por el estudio de los puntos salientes y de las lineamientos generales, vaya poco a poco entrando en conocimiento de los detalles. La enseñanza debía ser adecuada, la enseñanza llenaba esta condición cuando por su naturaleza y el sentido en que se daba tenía como fin que en cada periodo del niño y según las circunstancias de éste debía proseguirse. La enseñanza debía ser amena, agradable y atractiva, para esto se requería que el maestro fuera afable, benévolo, paciente de palabra fácil y elegante, vivo y animado, que sienta gusto en estar con los niños, en hacerse niño con ellos y en ponerse a su nivel ${ }^{30}$.

La enseñanza terminaba siendo un medio para conseguir dos fines básicos: la instrucción y la educación. La educación se centraba en el desarrollo intelectual del niño, constituyéndose en un elemento mucho más valioso que la instrucción. La instrucción podía colocar, depositar, amontonar conocimientos en la inteligencia, como un almacén y enriquecer la memoria con ellos a manera de provisiones que aportara a cierto tipo de desarrollo pasivo que esos conocimientos llevaban consigo, pero no con el vigor, la acción y la vivacidad que el entendimiento necesita. El fin de la enseñanza no era el saber, sino el ejercicio de la mente; no se trataba de transmitir conocimientos de literatura, historia, filosofía, sino de fortificar la imaginación, la memoria y el juicio, tarea que debía ser permanente en la escuela.

Por otra parte, la influencia del maestro en la escuela no se limitaba al niño, sino que se extendía a un gran número de familiares. El maestro se debía mostrar superior a la mayor parte de los padres de familia. El mejor maestro no podía

30 Martín Restrepo Mejía y Luis Restrepo Mejía, Elementos de Pedagogía, 175 
darle talentos al educando, sino desarrollarlos, florecerlos y fecundarlos. En Educación doméstica nos aclara que:

Tened muy presente que no basta que el maestro sepa las materias que ha de estudiar el niño. Es preciso que sepa enseñarla y esto rara vez se alcanza con dotes naturales: sin el estudio y la práctica de la pedagogía es casi imposible que una persona sepa enseñar. Hay mil secretos, mil detalles, mil reglas y principios para saber dirigir la inteligencia del niño de una manera tal que no sólo entienda lo que se le enseña, sino que se desarrolle ordenadamente, a fin de que no se vea abrumada por el estudio y confundida con el acopio de enseñanzas, antes bien, cada día se encuentra más capaz y vigorosa $a^{31}$.

El maestro debía dominar la enseñanza que, vista como una práctica, se constituía en el principal medio para la educación del entendimiento. La enseñanza como práctica, debía ser gradual y proporcional a la edad y las necesidades del niño; debía ser clara, cíclica y permanente. La enseñanza del maestro debía ser adecuada a las circunstancias y a los contextos; debía ser amena y agradable. La enseñanza, vista como una práctica bien manejada, le permitía al maestro lograr dos fines básicos: la educación del niño y su instrucción en el arte y la ciencia. De tal manera, el maestro se constituía como sujeto en la enseñanza, vista como práctica, como dominio de saber, en los marcos de dominio intelectual que le demandaban a este sujeto no solo saber la materia, sino saberla enseñar, lo que exigía el estudio del niño.

\section{Conceptos de educación escolar}

Los hermanos Restrepo Mejía, en Elementos de Pedagogía, establecen un concepto de educación basado en la transformación cualitativa del niño, acompañado de un tipo de institución acorde a su edad, que buscaba la final inserción de este sujeto a la sociedad, de acuerdo a ciertas variables y razones de carácter personal, social o económico. La educación debía buscar fines físicos, intelectuales y morales, pero también prácticos desde la perspectiva de Fröebel, que le permitieran la inserción de la sociedad a través de un oficio. De tal manera, se hacía preciso no confundir la educación con la instrucción, pero tampoco se podían considerar como dos cosas diversas o contrarias, debiéndose concebir como la parte de un todo. En sus palabras:

Instruir es educar, pero no es educar, sino parcialmente y cuando se persigue este fin descuidando los demás, no se alcanza el bien o se alcanza con detrimento de otros. La educación es la conducción del hombre a todos sus fines; mientras que la instrucción es la conducción del hombre a uno de ellos: al conocimiento ${ }^{32}$.

31 Martín Restrepo Mejía, Pedagogía doméstica. Autoeducación, dirección del hogar y educación de los hijos, 180.

32 Martín Restrepo Mejía y Luis Restrepo Mejía, Elementos de Pedagogía, 7 
El concepto de educación en Elementos de pedagogía, se basó en un alto porcentaje en preceptos católicos. Así, la educación sería ineficaz si no se realizaba de conformidad con las leyes del desarrollo humano, y si no se empleaban todos los medios necesarios. Así que el fin último del hombre, era la posesión de Dios y el último de la voluntad en la vida presente. Como ya se había evidenciado, para los hermanos Restrepo Mejía, en Elementos de Pedagogía, la virtud no la podía alcanzar una educación excesivamente racionalista, objetivista y apartada de Dios. El iusnaturalismo de Rousseau, ${ }^{33}$ debía situarse en un contexto nacional en el que este tipo de pedagogos precursores de la escuela nueva y las misiones religiosas, cuestionaron la doctrina sobre la naturaleza infantil, vista como peligrosa.

La educación se dividía, en primer lugar, en consiente e inconsciente: la educación consciente se daba intencionalmente, acompañada de un conjunto de medios e influencias que se ponían en juego para dirigir al niño; se podía realizar con un plan claro, según la educación que se buscara promover. El fin que proponía la pedagogía, era establecer los principios y reglas de la educación. Para los hermanos Restrepo Mejía, la educación era el objeto final de la pedagogía; la pedagogía era una ciencia antropológica, que abordaba al hombre. Ella se distinguía sobre las demás ciencias, porque las ponía a su servicio, colocando como fin último establecer los principios y leyes de desarrollo humano y de su conducción.

Para los hermanos Restrepo Mejía, debía existir una relación directa entre la educación doméstica y la educación escolar, entre la educación inconsciente y la educación consciente. La educación doméstica tenía como ventajas el conservar y fortalecer los afectos de familia; el niño daba alegría, unión y felicidad a sus padres y desterraba del hogar los vicios, porque su presencia exigía cuidados y ejemplo. El niño educado en su casa comprendía la importancia de la familia y debía aprender cómo debía gobernarse la casa, administrarse los intereses familiares, educar a los hijos y cumplir con todos los deberes domésticos.

El hijo, en el interior de su hogar, seguía en un alto porcentaje del tiempo al lado de los padres; por lo tanto, de ellos recibía el principal ejemplo con las conversaciones de la familia; era influenciado por medio de las costumbres familiares, y en la educación doméstica, el niño recibía principios de moral profundos que no podía garantizar la escuela. Inclusive, la educación doméstica bien estructurada, podía ofrecer una educación intelectual más práctica y una educación física más definida. Desde la perspectiva de la educación doméstica,

33 La tensión entre los pedagogos y educadores católicos colombianos, frente a la obra de J.J. Rousseau, como lo da a conocer Sáenz, Saldarriaga y Ospina, en Mirar la Infancia, se mantuvo hasta los años cuarenta del siglo XX. En buena medida, los jesuitas, quienes eran muy cercanos a Martín Restrepo Mejía, por medio de la Revista Razón y Fe, descalificaron continuamente la obra de Rousseau y de paso varios preceptos epistemológicos y conceptuales de la escuela activa (Consultar: Javier Sáenz, Oscar Saldarriaga y Armando Ospina, Mirar la infancia. Pedagogía, moral y modernidad en Colombia, 1903-1946, Bogotá, D.C., Colciencias, Vol. 1, 1997, 111). Una tensión similar, para esa época, se vive en el Departamento de Antioquia, cuando Luis Carlos Tejada Cano (1898-1924), sustentó su trabajo de grado en la Escuela Normal de Medellín, en 1916, defendiendo los preceptos educativos de Rousseau y la escuela activa, terminado expulsado por parte de los Hermanos Cristianos, viendo así frustrada su formación como maestro, que era su proyecto de vida inicial, para dedicarse, luego, a la crónica periodista moderna en el diario El Espectador, empresa en la que tuvo gran éxito (Consultar: Lino Gil Jaramillo, Tripulantes de un barco de papel, Medellín, Editorial Beta, 1975). 
les correspondía a los padres dirigir al niño, entre los siete y catorce años, y ante todo, preguntarse cómo completar la labor del maestro, a fin de que la educación del niño resultara de la acción armónica de los padres.

En la familia, los padres podían conocer mejor a sus hijos, conservar su inocencia y educarlos con interés y eficacia. Pero la educación doméstica no bastaba, porque los padres carecían, por lo general, de tiempo, de las aptitudes y los conocimientos necesarios para educar a sus hijos. A la educación doméstica debía agregársele la escolar, debido a que la escuela ponía al alcance una cantidad inestimable de bienes de la educación. La escuela ponía a los niños en mutuo contacto y los interesaba en un mismo trabajo, los estimulaba, los ponía a trabajar con ahínco y placer. La disciplina escolar se mostraba como superior a la doméstica; la influencia del maestro era poderosísima, y el niño cumplía mejor sus deberes en la escuela que en el hogar.

De otro lado, de acuerdo a las transformaciones filosóficas dadas en la modernidad, los hermanos Restrepo Mejía, clasificaron los sistemas de educación en términos filosóficos, en materialista y espiritualista, ${ }^{34}$ y en términos de propuestas prácticas, en sistemas racionalistas y cristianos. Para los positivistas, la educación se constituía en el arte de desarrollar continuamente la especie, el individuo y sus potencias. Desde la perspectiva de Rousseau, la educación se centraba en el desarrollo de las facultades del hombre, y para Pestalozzi, se expresaba en el completo desarrollo de las "facultades humanas" 35 para convertir al niño en un hombre. Los racionalistas se basaban, de manera central, en las leyes del desarrollo humano, acompañados de métodos conforme a la naturaleza del hombre. Sin embargo, la pedagogía moderna, propuesta por Martín Restrepo Mejía, debía a la doctrina cristiana el verdadero sistema de educación, un conjunto de principios, leyes y métodos inmejorables que la escuela moderna no podía desconocer.

La educación escolar demandaba un apoyo institucional del Estado, que se inscribía en un concepto moderno de escuela, que hacía parte de una red institucional que debía pensar en el niño desde su etapa de parvulario, hasta su adolescencia y juventud. La educación escolar debía buscar fines físicos, intelectuales y morales en torno al niño, sin confundir su esencia y filosofía con la simple instrucción. La educación, siendo el objeto final de la pedagogía, buscaba

34 El primero, el materialista, con sus dos vertientes: indefinido y sensualitas, que ponía como fin último la vida presente, el perfeccionamiento continuo de la especie humana y del individuo, establecía además que el hombre en su origen fue un animal y que no se distinguía de los demás en el grado de desarrollo alcanzado; el perfeccionamiento humano se basa en fines temporales y la muerte pone fin a la existencia. El principal vocero de este sistema es Herbert Spencer. También, este pone énfasis en el goce sensible, obteniendo la mayor suma posible de placeres, ubicando aquí también a Locke y Hobbes. El segundo, el espiritualista, reconoce en el hombre un espíritu como una manifestación de Dios, en este se pueden ubicar los lineamientos pedagógicos de Froebel y el mismo Kant, que ponían fin del desarrollo en la sabiduría, en el hacer el bien y en la belleza humana. En el sistema espiritualista, de carácter egoísta o individual, se encuentra la iniciativa pedagógica de J.J. Rousseau en Emilio, quien estableció que el hombre es bueno y la sociedad lo corrompe, siguiendo sus pasos se ubica la pedagogía pestalozziana.

35 Martín Restrepo Mejía, centró su propuesta educativa en el desarrollo de las "facultades humanas", la cual era una posición intermedia entre las facultades del alma, que se adscribían claramente a una perspectiva religiosa y las facultades mentales que podían excluir toda referencia a la sustancia espiritual (Consultar: Sáenz, Saldarriaga y Ospina, Mirar la infancia. Pedagogía, moral y modernidad en Colombia, 1903-1946, Vol. 2, 136). Nuevamente, pero ahora en términos de filosofía educativa, encontramos a este pedagogo en una situación de "liminidad", buscando negociar las relaciones de tensión entre fe y razón, pero ahora en un momento de transición educativa en el que los preceptos de la escuela activa entraban con potencia en la Colombia de los años de 1920. 
el desarrollo de las "facultades humanas" que, como punto intermedio entre espíritu y razón, formaba desde esta propuesta educativa, seres integrales y, por qué no decirlo, hombres preparados para el cambio de época que se avecinaba.

\section{CONCLUSIÓN}

Al abordar en retrospectiva la vida y obra de Martín Restrepo Mejía, debemos reconocer que, a pesar de su adscripción al partido conservador y su relación con la Iglesia católica, fue un maestro e intelectual de la educación que modernizó el pensamiento pedagógico colombiano desde finales del siglo XIX y, por lo menos, hasta 1920. Su incidencia en la pedagogía colombiana es insoslayable ya sea en lo que respecta a la manera como asume la reflexión científica de la educación, ya sea por su concepción de infancia y de maestro, ya sea por su incidencia en las Escuelas Normales en las que se formaron los maestros de la época en mención. Lo anterior, por medio de su obra cumbre, escrita con su desaparecido hermano Luis, Elementos de Pedagogía, libro que, por lo demás, tuvo una resonancia importante en su época debido a que se constituyó en el principal manual para la formación de maestros en las escuelas normales que existían en el país, acompañadas de sus escuelas anexas. La modernización de la pedagogía colombiana conllevó la lectura de pedagogos, como Pestalozzi, Froebel, Montessori y Decroly, acompañada de una lectura crítica de Rousseau, para adaptar sus propuestas a la situación educativa nacional, lo que conllevó la individualización del niño y pensar los temas de la infancia moderna.

La pedagogía moderna se constituyó en un proceso de transformación de lo que era la "educación memorista" o método tradicional, en el que habían sido formados los maestros colombianos y en el que se ubicaba un concepto de escuela decimonónico. Dicha realidad, Martín Restrepo Mejía la quiso transformar a través de sus reflexiones pedagógicas, su labor didáctica y elaboración de manuales escolares sobre la matemática, la lengua castellana, la historia universal, la educación doméstica y la pedagogía del parvulario, entre otras. Cuando valoramos parte de su pensamiento pedagógico y desglosamos sus conceptos de pedagogía, infancia, maestro, educación escolar, evidenciamos un momento de apropiación y transición entre la reforma educativa liberal de 1870 y la aparición de la escuela activa en Colombia, que se dio en 1914, con la fundación del Gimnasio Moderno, en la ciudad de Bogotá.

Martín Restrepo Mejía, como intelectual de la educación y precursor católico de la escuela nueva, representa una transición de carácter modernizante entre una pedagogía cristiana que, en términos filantrópicos, demandó el amor hacia la labor de enseñanza, que era la labor propia del maestro; y la pedagogía moderna, la pedagogía objetiva, que establecía otros fines ontológicos y políticos para transformar al sujeto, la escuela y la sociedad a lo largo del siglo XX. En virtud de lo anterior, rescatar el pensamiento pedagógico de este tipo de intelectuales 
de la educación y valorarlos en su contexto histórico, se constituye en una tarea que aporta a la consolidación de la identidad pedagógica colombiana.

Vista de manera retrospectiva la obra de Martín Restrepo Mejía fue una de las primeras bases para la constitución del Campo intelectual de la pedagogía en Colombia, que responde también a una serie de acontecimientos a tener en cuenta, como la primera reforma educativa de 1870, el plan Zerda de 1893, la primera ley Orgánica de Educación conocida como la Ley 39 de 1903, la realización del Primer Congreso Pedagógico Nacional de 1917, la fundación y funcionamiento de la Escuela Normal Superior de Colombia,-entre 1936 y 1952-, la fundación de las Universidades Pedagógicas en Bogotá y Tunja, la consolidación de las Facultades de Educación a mediados del siglo XX, y entre otras la actual Ley General de Educación Ley 115 de 1994. De tal manera, nuestros maestros y pedagogos más destacados, vistos como intelectuales de la educación, se encuentran haciendo parte de este trasfondo en un país caracterizado, hasta épocas recientes, por prácticas verticales, conservadoras y muy católicas, en la toma de decisiones, tanto como por intentos de democratización, liberalización, secularización e incluso de cambio y revolución en nuestro sistema educativo.

\section{FUENTES}

Restrepo Mejía, Martín, y Restrepo Mejía, Luis. Elementos de Pedagogía. Bogotá: Imprenta Eléctrica, 1905.

Restrepo Mejía, Martín. Labor didáctica. Bogotá: Imprenta Moderna, 1909.

Restrepo Mejía, Martín. Pedagogía doméstica. Autoeducación, dirección del hogar y educación de los hijos. Bogotá: Editorial Barcelona, 1914.

Restrepo Mejía, Martín. Pedagogía de párvulos. Exposición de la enseñanza activa. Bogotá: Editorial de Cromos, ca.1925.

\section{REFERENCIAS}

Bohórquez Casallas, Luis Antonio. Evolución Educativa en Colombia, Bogotá: Publicaciones Cultural Colombiana, 1956.

Foucault, Michel. Historia de la sexualidad I. La voluntad de saber. México: Siglo XXI, 1976. Franco Ferrarotti, "Las Historias de Vida como Método", Convergencia Vol. 14, No. 44 (2007)

Gennep, van Arnold. Los ritos de Paso. Madrid: Alianza Editorial, 2008.

Gil Jaramillo, Lino. Tripulantes de un barco de papel. Medellín: Editorial Beta, 1975.

Jiménez Becerra, Absalón. El campo de la pedagogía y otros ensayos sobre la historia de infancia en Colombia. Bogotá, D.C.: UDFJC, 2014. 
Jiménez Becerra, Absalón. “I Congreso Pedagógico Nacional Colombiano de 1917. Una mirada a sus tensiones y avances, cien años después de su realización". (Revista Pedagogía y Saberes, No. 48. Bogotá, D.C. CIUP-UPN).

Jiménez B., Absalón. "Algunos elementos para la investigación en historia”. En Absalón Jiménez Becerra y Alfonso Torres Carrillo: La práctica investigativa en Ciencias Sociales Colombia. Bogotá, D.C.: UPN, 2004.

Mora, José Pascual; Soto Arango, Diana Elvira; Lima Jardilino, José Rubens. “La historia de la educación en América Latina: la contribución y aportes de la Sociedad de historia de la educación latinoamericana" (1994-2014). História da Educação, Vol. 21 No. 51 (2017) 351-375. https://dx.doi.org/10.1590/2236-3459/66357.

Paternina Soto, Liliana. "Selección bibliográfica sobre Escuela Nueva en Colombia" Revista Historia de la Educación Latinoamericana No. 5. (1998): 144 - 148.

Saldarriaga Vélez, Oscar. Del oficio de Maestro. Práctica y teorías de la pedagogía moderna en Colombia. Bogotá, D.C.: Cooperativa Editorial Magisterio, Grupo de Historia de la Práctica Pedagógica, 2003.

Sáenz, Javier; Saldarriaga, Oscar y Ospina Armando. Mirar la infancia. Pedagogía, moral y modernidad en Colombia, 1903-1946. Bogotá, D.C.: Colciencias, 1997.

Soto Arango, Diana Elvira. La Escuela Rural en Colombia. Historias de Vida de Maestras. Mediados del Siglo XX. Tomo VI. Colección Educadores, (Tunja, Colombia, 2014).

\begin{tabular}{l|l} 
& \\
\hline & Jiménez Becerra, Absalón. “Pensamiento pedagógico colombiano: \\
Martín Restrepo Mejía. Una mirada a sus conceptos de pedagogía, & \\
infancia, maestro y escuela”. Revista Historia de la Educación & \\
Latinoamericana. Vol. 19 No. 29 (2017): 245-269. & \\
\hline &
\end{tabular}

